\title{
PLASMA NEUTRALIZATION MODELS FOR INTENSE ION BEAM TRANSPORT IN PLASMA
}

\author{
Igor D. Kaganovich, Edward A. Startsev, Ronald C. Davidson and Sean O'Rourke \\ Plasma Physics Laboratory, Princeton University, Princeton, New Jersey 08543 \\ Edward P. Lee, Lawrence Berkeley National Laboratory, Berkeley, California 94720
}

\begin{abstract}
Plasma neutralization of an intense ion pulse is of interest for many applications, including plasma lenses, heavy ion fusion, cosmic ray propagation, etc. An analytical electron fluid model has been developed based on the assumption of long charge bunches $\left(l_{b}>>r_{b}\right)$. Theoretical predictions are compared with the results of calculations utilizing a particle-in-cell (PIC) code. The cold electron fluid results agree well with the PIC simulations for ion beam propagation through a background plasma. The analytical predictions for the degree of ion beam charge and current neutralization also agree well with the results of the numerical simulations. The model predicts very good charge neutralization $(>99 \%)$ during quasi-steady-state propagation, provided the beam pulse duration $\tau_{b}$ is much longer than the electron plasma period $2 \pi / \omega_{p}$, where $\omega_{p}=\left(4 \pi e^{2} n_{p} / m\right)^{1 / 2}$ is the electron plasma frequency, and $n_{p}$ is the background plasma density. In the opposite limit, the beam pulse excites large-amplitude plasma waves. The analytical formulas derived in this paper can provide an important benchmark for numerical codes, and provide scaling relations for different beam and plasma parameters.
\end{abstract}

\section{INTRODUCTION}

Neutralization of the ion beam charge and current by a background plasma is an important issue for many applications involving the transport of positive charges in plasma, including heavy ion inertial fusion, positrons for electron-positrons colliders, high-density laser-produced proton beams for the fast ignition of inertial confinement fusion targets, etc.

There are many critical parameters for ion beam transport in the target chamber, including beam current, type of ion species, radial and longitudinal profiles of the beam density, chamber gas density, stripping and ionization cross sections, etc. This necessitates an extensive study for a wide range of parameters to determine the conditions for optimum beam propagation. To complement numerical simulation studies, a number of reduced models have been developed. Based on wellverified assumptions, reduced models can yield robust analytical and numerical descriptions and provide important scaling laws for the degrees of charge and current neutralization.

The electron response frequency is of order the electron plasma frequency, $\omega_{p}=\left(4 \pi n_{p} e^{2} / m_{e}\right)^{1 / 2}$, where $n_{p}$ is the background plasma density. For heavy ion fusion applications, the ion pulse propagation time through the chamber is much longer than the inverse electron plasma frequency $\omega_{p}^{-1}$. Therefore, a beamplasma quasi-steady state forms during beam propagation. The initial step of the study is to describe the steady-state propagation (in the beam frame) of an ion beam pulse through a background plasma.

The case where the beam propagates through a cold plasma, with the plasma density large compared with the beam density, can be studied by the use of linear perturbation theory [1]. Here, we focus on the nonlinear case where the plasma density has an arbitrary value compared with the beam density, and correspondingly, the degrees of current and charge neutralization are arbitrary. The transport of stripped, pinched ion beams has also been discussed in [2], where the assumptions of current and charge neutrality were made to determine selfconsistent solutions for the electric and magnetic fields. Rosenbluth, et al., have considered the equilibrium of an isolated, charge-neutralized, self-pinched ion beam pulse in the absence of background plasma [3]. In contrast, we consider here the case where "fresh" plasma is always available in front of the beam, and there are no electrons co-moving with the beam.

In a recent calculation [4,5], we studied the nonlinear quasi-equilibrium properties of an intense ion beam pulse propagating through a cold background plasma, assuming that the beam pulse duration $\tau_{b}$ is much longer than the inverse electron plasma frequency, i.e., $\omega_{p} \tau_{b} \gg 1$. In the present study, we generalize the previous results to general values of the parameter $\omega_{p} \tau_{b}$.

\section{BASIC EQUATIONS FOR ION BEAM PULSE PROPAGATION IN BACKGROUND PLASMA}

In most applications, the background plasma electrons are cold - the electron thermal velocity is small compared with the directed beam velocity. Particle-in-cell simulations show that in most cases the electron flow is laminar and does not form multistreaming. Thus, the electron cold-fluid equations can be used for the electron description, and thermal effects are neglected in the present study. The electron fluid equations together with Maxwell's equations comprise a complete system of equations describing the electron response to a propagating ion beam pulse. The electron cold-fluid equations consist of the continuity equation,

$\frac{\partial n_{e}}{\partial t}+\nabla \cdot\left(n_{e} \mathbf{V}_{e}\right)=0$ 
and the force balance equation,

$\frac{\partial \mathbf{p}_{e}}{\partial t}+\left(\mathbf{V}_{e} \cdot \nabla\right) \mathbf{p}_{e}=-e\left(\mathbf{E}+\frac{1}{c} \mathbf{V}_{e} \times \mathbf{B}\right)$,

where $-e$ is the electron charge, $\mathbf{V}_{\mathbf{e}}$ is the electron flow velocity, $\mathbf{p}_{e}=\gamma_{e} m_{e} \mathbf{V}_{e}$ is the average electron momentum, $m_{e}$ is the electron rest mass, and $\gamma_{e}$ is the relativistic mass factor. Maxwell's equations for the self-generated electric and magnetic fields, $\mathbf{E}$ and $\mathbf{B}$, are given by

$\nabla \times \mathbf{B}=\frac{4 \pi e}{c}\left(Z_{b} n_{b} \mathbf{V}_{b}-n_{e} \mathbf{V}_{e}\right)+\frac{1}{c} \frac{\partial \mathbf{E}}{\partial t}$,

$\nabla \times \mathbf{E}=-\frac{1}{c} \frac{\partial \mathbf{B}}{\partial t}$,

where $\mathbf{V}_{\mathbf{b}}$ is the ion beam flow velocity, $n_{e}$ and $n_{b}$ are the number densities of the plasma electrons and beam ions, respectively (far a way from the beam $n_{e} \rightarrow n_{p}$ ), and $Z_{b}$ is the ion beam charge state. The plasma ions are assumed to remain stationary with $\boldsymbol{V}_{\boldsymbol{i}}=0$ and $n_{i}=n_{p}$. The assumption of immobile plasma ions is valid for sufficiently short ion pulses with $2 l_{b}<r_{b} \sqrt{M / m_{e}}$ [4]. Here, $r_{b}$ and $2 l_{b}$ are the ion beam radius and length, respectively, and $M$ is the plasma ion mass.

Considerable simplification can be achieved by applying the conservation of generalized vorticity $\boldsymbol{\Omega}$ [4]. If $\boldsymbol{\Omega}$ is initially equal to zero ahead of the beam, and all streamlines inside of the beam originate from the region ahead of the beam, then $\boldsymbol{\Omega}$ remains equal to zero everywhere, i.e.,

$\mathbf{\Omega} \equiv \nabla \times \mathbf{p}_{e}-\frac{e}{c} \mathbf{B}=0$.

Substituting Eq.(5) into Eq.(2) yields

$\frac{\partial \mathbf{p}_{e}}{\partial t}+\nabla K_{e}=-e \mathbf{E}$

where $K_{e}=\left(\gamma_{e}-1\right) m_{e} c^{2}$ is the electron kinetic energy. Note that the inertia terms in Eq.(6) are comparable in size to the Lorentz force term and cannot be omitted. Estimating the self-magnetic field from Eq.(5), we conclude that the electron gyroradius is of order the beam radius. This is a consequence of the fact that the electrons originate from the region of zero magnetic field in front of the beam. If most electrons are dragged along with the beam and originate from the region of large magnetic field, the situation may be different $[3,6]$.

\section{APPROXIMATE SYSTEM OF EQUATIONS FOR LONG CHARGE BUNCHES $\left(l_{b} \gg r_{b}\right)$}

We use the assumption of a long ion pulse $\left(l_{b} \gg r_{b}\right)$, but relax the assumption of a dense beam used in [4,5], i.e., the condition $V_{b} / \omega_{p}<l_{b}$.

The typical longitudinal scale of electron density perturbations is $V_{b} / \omega_{p}$. If $V_{b} / \omega_{p} \gg r_{b}$, the main variations are in the radial direction, and longitudinal derivatives can be neglected in comparison with the radial derivatives in Poisson's equation. This gives for steadystate beam propagation

$\left(V_{e z}-V_{b z}\right) \frac{\partial n_{e}}{\partial z}+\frac{1}{r} \frac{\partial}{\partial r}\left(r n_{e} V_{e r}\right)=0$,

$\frac{1}{r} \frac{\partial}{\partial r}\left(r E_{r}\right)=4 \pi e\left(Z_{b} n_{b}+n_{p}-n_{e}\right)$,

$\left(V_{e z}-V_{b z}\right) \frac{\partial}{\partial z} p_{e r}+\frac{\partial K_{e}}{\partial r}=-e E_{r}$.

It follows from Eq.(5) for cylindrically symmetric beams that the azimuthal self-magnetic field is determined in terms of the longitudinal flow velocity, which gives

$$
\begin{aligned}
& B=-\frac{c}{e} \frac{\partial p_{e z}}{\partial r}, \\
& -\frac{1}{r} \frac{\partial}{\partial r} r \frac{\partial}{\partial r} p_{e z}=\frac{4 \pi e}{c}\left(Z_{b} n_{b} V_{b z}-n_{e} V_{e z}\right) .
\end{aligned}
$$

Equation (11) describes the longitudinal electron flow velocity and determines the degree of current neutralization of the beam. In Eq. (11), we neglected the displacement current. The displacement current can be comparable with the electron current if $V_{b} / \omega_{p}$ is comparable with $l_{b}$. However, in this case both the displacement current and the electron current are small compared with the other terms in Eq. (11).

Numerical solutions of the system of equations consisting of Eqs. (7) - (9) and Eq.(11) are presented in [7] and [8]. In Fig.1, we present a detailed comparison of the fluid and PIC results. The fluid results of Eqs. (7) - (9) and Eq. (11) agree well with the results of the twodimensional electromagnetic PIC simulations described in [4].

In the linear case, when $n_{p} \gg Z_{b} n_{b}$, the equation for electron plasma oscillations is given by [4]

$$
V_{b z}^{2} \frac{\partial^{2}}{\partial z^{2}} n_{e}+\omega_{p}^{2}\left(n_{e}-Z_{b} n_{b}-n_{b}\right)=0 .
$$

Equation (12) is readily recovered from the linearized version of Eqs.(1) and (2) and Poisson's equation, and is not restricted by any requirements on the beam radius. It can also be derived from Eqs. (7) - (9) when the nonlinear terms are neglected. Because the reduced model consisting of Eqs. (7) - (9) and Eq. (11) gives the same results in the limit $V_{b} / \omega_{p} \gg r_{b}$, and in the linear case where $n_{p} \gg Z_{b} n_{b}$, it also works well in the intermediate case where $V_{b} / \omega_{p} \sim r_{b}$, as can be seen in Fig.1. For $n_{b}>n_{p}$, the plasma waves break [4] as shown in Figure 2. The utilization of the cold-fluid model is therefore limited by the wave-breaking condition.

The approach used here can be generalized to the case of a nonuniform, nonstationary, warm electron fluid, and forms the basis for a hybrid, semi-analytical approach to be used for calculations of beam propagation in the target chamber. This research is now underway.

In summary, the analytical results agree well with the results of PIC numerical simulations for ion beam charge and current neutralization. The visualization of the data 

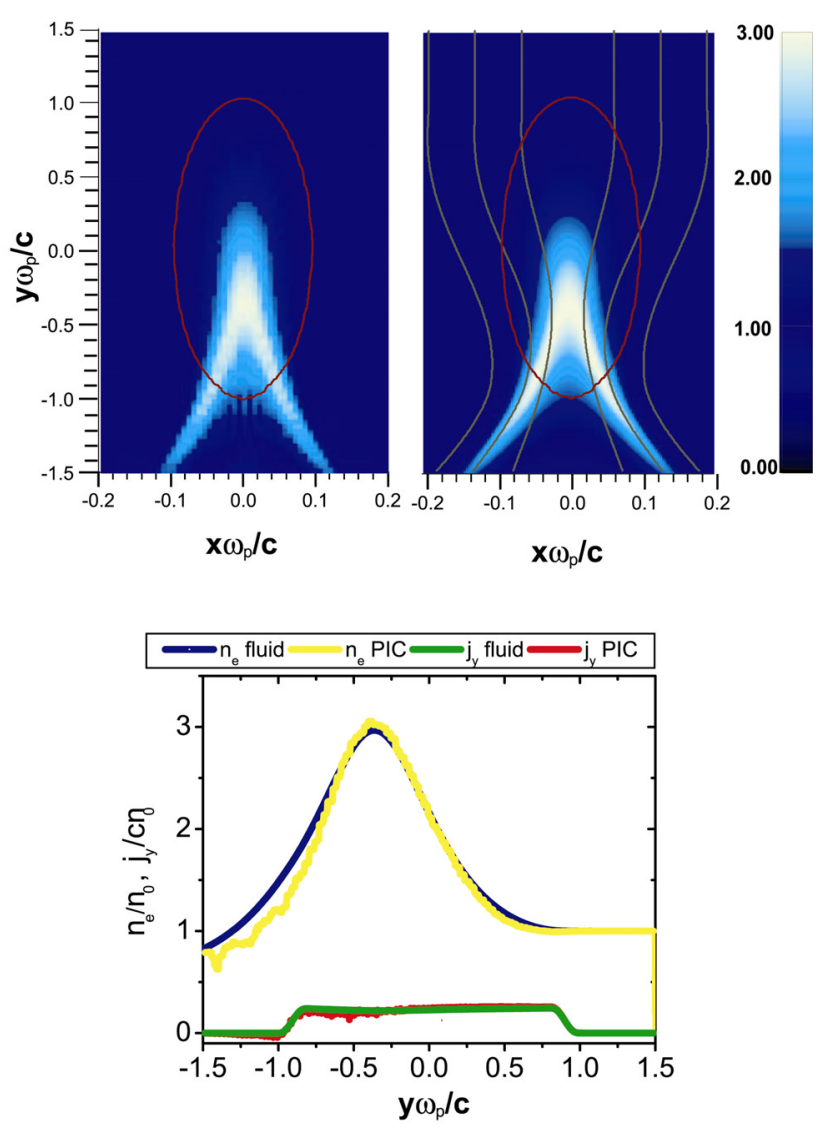

Figure 1 Neutralization of an ion beam pulse during steady-state propagation of the beam pulse through a cold, uniform, background plasma. The figure shows comparisons between the PIC simulations and the fluid description. The beam propagates in the y-direction. The beam density has a flat-top profile, and the red lines show the beam pulse edge. Shown in the figure are color plots of the normalized electron density $\left(n_{e} / n_{p}\right)$ for particlein-cell simulations (top left) and the fluid model consisting of Eqs. (7) - (9) and Eq. (11) (top right) in $\left(x \omega_{p} / c, y \omega_{p} / c\right)$ space. The lower figure shows the normalized electron density $\left(n_{e} / n_{p}\right)$, and the normalized longitudinal current $\left(j_{y} / e n_{p} c\right)$ in the beam cross-section at $\mathrm{x}=0$ (lowest curves). The brown contours in the upper figure show the electron trajectories in the beam frame. The beam velocity is $V_{b}=0.5 c$, and the beam density is $n_{b}=0.5 n_{p}$. The beam dimensions correspond to $r_{b}=0.1 c / \omega_{p}$ and $l_{b}=1.0 \mathrm{c} / \omega_{p}$.

obtained in the numerical simulations shows complex collective phenomena during beam entry into and exit from the plasma, and will be described in future publications. Further visualization is also available on the website [9].

Acknowledgments: This research was supported by the U.S. Department of Energy Office of Fusion Energy Sciences and the Division of High Energy Physics.
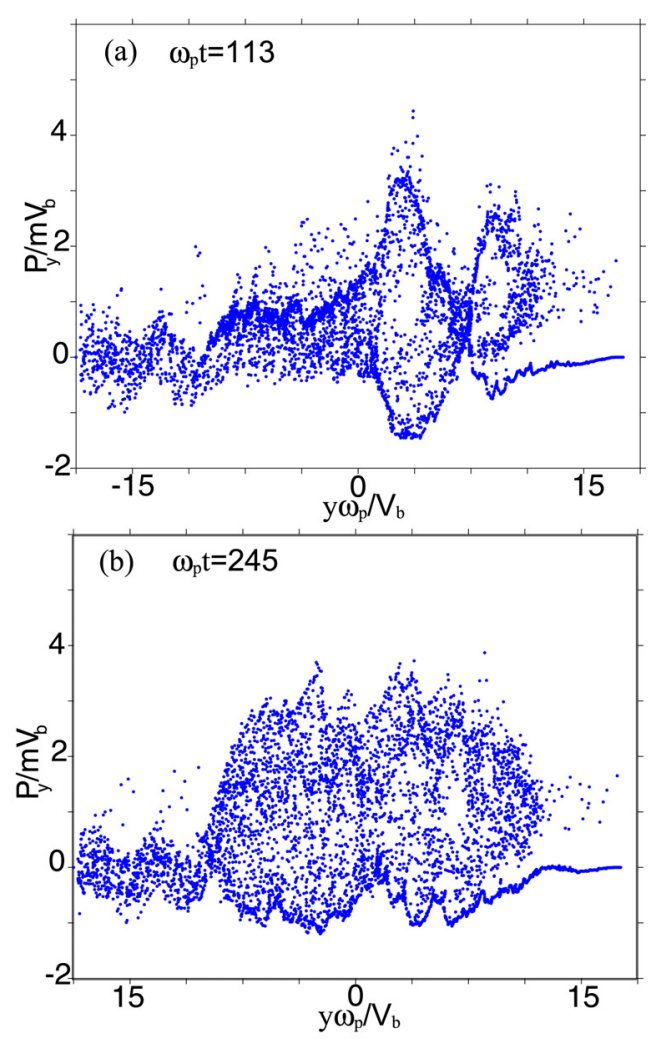

Figure 2 Electron phase space for 1D simulation of beam entering the plasma at $\mathrm{t}=0$. Here, $l_{b}=30 V_{b} / \omega_{p}$ and $n_{b}=2 n_{p}$. The times after entering the plasma plug correspond to (a) $\mathrm{t}=113 / \omega_{p}$, and (b) $\mathrm{t}=245 / \omega_{\mathrm{p}}$.

\section{REFERENCES}

[1] P. Chen, J.M. Dawson, R. W. Huff and T. Katsouleas, Phys. Rev. Lett. 51, 693 (1985).

[2] K. Hahn and E. P. Lee, J. Fusion Engineering and Design 32-33, 417 (1996).

[3] M. Rosenbluth, E.P. Lee and R. Briggs, private communication (2001).

[4] I. D. Kaganovich, G. Shvets, E. Startsev and R. C. Davidson, Physics of Plasmas 8, 4180 (2001).

[5] I. D. Kaganovich, E. Startsev and R. C. Davidson, Laser and Particle Beams 20, 497 (2002).

[6] D. R. Welch, D. V. Rose, B. V. Oliver, T. C. Genoni, R. E. Clark, C. L. Olson and S. S. Yu, Phys. Plasmas 9, 2344 (2002).

[7] I. D. Kaganovich, E. Startsev, S. Klasky and R. C. Davidson, Special Issue on Images in Plasma Science, IEEE Trans. Plasma Science 30, 12 (2002).

[8] I. D. Kaganovich, G. Shvets, E. Startsev and R. C. Davidson, Proc. 2001 Particle Accelerator Conference; $\quad$ http://pacwebserver.fnal.gov/papers/ Tuesday/PM_Poster/TPPH317.pdf.

[9] High Intensity Particle Beams and Nonneutral Plasma, Division, Plasma Physics Laboratory, Princeton University; http://w3.pppl.gov/ nnp/. 